\title{
BMJ Open PREventive effect of FENestration with and without clipping on post-kidney transplantation lymphatic complications (PREFEN): study protocol for a randomised controlled trial
}

Mohammad Golriz, ${ }^{1}$ Mohammadsadegh Sabagh, ${ }^{1}$ Sara Mohammadi, ${ }^{1}$ Omid Ghamarnejad, ${ }^{1}$ Elias Khajeh, ${ }^{1}$ Markus Mieth, ${ }^{1}$ Mohammed Al-Saeedi, ${ }^{1}$ Markus K Diener, ${ }^{1}$ André L Mihaljevic, ${ }^{1}$ Christian Morath, ${ }^{2}$ Martin Zeier, ${ }^{2}$ Yakup Kulu, ${ }^{1}$ Arianeb Mehrabi (D) ${ }^{1}$

To cite: Golriz M, Sabagh M, Mohammadi S, et al. PREventive effect of FENestration with and without clipping on post-kidney transplantation lymphatic complications (PREFEN): study protocol for a randomised controlled trial. BMJ Open 2020;10:e032286. doi:10.1136/ bmjopen-2019-032286

- Prepublication history for this paper is available online. To view these files, please visit the journal online (http://dx.doi. org/10.1136/bmjopen-2019032286).

Received 25 June 2019 Revised 18 February 2020 Accepted 20 August 2020

Check for updates

(C) Author(s) (or their employer(s)) 2020. Re-use permitted under CC BY-NC. No commercial re-use. See rights and permissions. Published by BMJ.

${ }^{1}$ Department of General, Visceral and Transplantation Surgery, University of Heidelberg, Heidelberg, Baden-Württemberg, Germany

${ }^{2}$ Department of Nephrology, Heidelberg University Hospital, Heidelberg, Germany

Correspondence to

Professor Dr. med. Arianeb Mehrabi;

arianeb.mehrabi@med.uniheidelberg.de

\section{ABSTRACT}

Introduction Peritoneal fenestration is an effective preventive method for reducing the rate of lymphatic complications in kidney transplantation (KTx). The size of the fenestration plays an important role in its effectiveness. A large peritoneal window is no longer indicated, due to herniation and difficulties in performing biopsies. Small preventive fenestration is effective but will be closed too early. The aim of this study is to evaluate whether metal clips around the edges of a small fenestration result in optimal effects with minimum fenestration size.

Methods and analysis This trial has been initiated in July 2019 and is expected to last for 2 and a half years. All patients older than 18 years, who receive kidneys from deceased donors, will be included. The kidney recipients will be randomly allocated to either a control arm (small fenestration alone) or an intervention arm (small fenestration with clipping). All fenestrations will be round, maximum $2 \mathrm{~cm}$, and close to the kidney hilum. Clipping will be performed with eight metal clips around the peritoneal window $\left(360^{\circ}\right)$ in every $45^{\circ}$ in an oblique position. The primary endpoint is the incidence of symptomatic post-KTx lymphatic complications, which require interventional treatment within 6 months after KTx. Secondary endpoints are intraoperative and postoperative outcomes, including blood loss, operation time, severity grade of lymphocele/lymphorrhea and relative symptoms. Ethics and dissemination This protocol study received approval from the Ethics Committee of the University of Heidelberg (Registration Number S-318/2017). A Standard Protocol Items: Recommendations for Interventional Trials checklist is available for this protocol. The results will be disseminated through peer-reviewed journals and conference presentations.

Trial registration number ClinicalTrials.gov Registry (NCT03682627).

\section{INTRODUCTION}

Lymphoceles and lymphorrhea are the most common complications after kidney
Strengths and limitations of this study

- The PREFEN Study is a double-blinded exploratory randomised controlled trial.

- PREFEN Study will be the first randomised controlled trial to investigate the impact of clipping the edges of a small peritoneal fenestration on lymphatic complications after kidney transplantation.

- The incidence of lymphocele and/or lymphorrhea will be investigated in the 6 months following kidney transplantation.

- Intraoperative easy flow drain will be placed in all participants, which may lead to different findings compared with other centres that do not routinely use drain.

- The findings of this trial may help to decrease the rate of lymphatic complications, as well as graft failure, hospitalisation time and costs.

transplantation $(\mathrm{KTx}){ }^{1-5}$ Incidence rates of post-KTx lymphoceles are high despite improvements in surgical methods and they are usually diagnosed during 2 weeks to 6 months after surgery. ${ }^{6}$ Post-KTx lymphoceles are usually asymptomatic and are identified during routine ultrasound examination. However, they may result in morbidities such as abdominal discomfort, impaired wound healing, thrombosis and even graft loss due to pressure on the kidney, the ureter or vascular structure. ${ }^{27}$ Because of the frequency and consequences of post-KTx lymphoceles, different preventive methods have been proposed in the literature ${ }^{13}$ such as: precise ligation of donor and recipient lymphatic vessels, ${ }^{2}$ drains at the site of graft implantation, ${ }^{8}$ polymeric sealants/haemostatic biomaterials, ${ }^{10}$ povidone-iodine, ${ }^{11}$ compression 
of the lower limb after KTx, ${ }^{12}$ intraoperative fluorescent lymphography ${ }^{13}$ and intraoperative bipolar cautery of lymphatic vessels. ${ }^{14}$ However, post-KTx lymphocele formation still remains a challenging complication that requires long-term postoperative interventions. ${ }^{1516}$

Peritoneal fenestration at the time of KTx is another simple method to prevent lymphocele formation. Although this method has been widely studied as a treatment of lymphoceles following KTx,${ }^{3}{ }^{17-19}$ to the best of our knowledge, only one randomised controlled trial (RCT) has investigated its impact on the incidence of post-KTx lymphocele. ${ }^{20}$ In this clinical trial, a large fenestration in the peritoneum (at least as long as the length of the implanted kidney) could reduce the lymphocele rate. The authors reported more intestinal complications following a large window. ${ }^{20}$ It is believed that a large peritoneal fenestration increases the rate of intestinal herniation and also makes it difficult to perform a renal biopsy. On the other hand, a smaller window in the peritoneal cavity increases the risk of early closure and subsequent lymphocele formation. ${ }^{21}$ Whether clipping the edges of a small peritoneal fenestration can prevent early closure, while preserving its prophylactic effect, has not yet been investigated.

The aim of this RCT is to investigate the rate of postkidney transplantation lymphatic complications (PKTL) in two groups of KTx patients: (1) with small fenestration only and (2) with small fenestration and clipping of its edges.

\section{METHODS AND ANALYSIS \\ Setting}

This is a double-blinded exploratory RCT. The trial will be performed at the Division of Transplantation Surgery, Department of General, Visceral and Transplantation Surgery, University of Heidelberg. This trial will be initiated in July 2019 and it is expected to progress for 2 and a half years.

\section{Patient recruitment}

The protocol of this study was reviewed and accepted by the independent Ethics Committee of the University of Heidelberg (registration number: S-318/2017, online supplemental file 1 ). The process of patient recruitment is shown in figure 1 . All recipients from deceased kidney donors, including left and right kidneys, will be screened for eligibility. Recipients who are not able to comply with study and/or follow-up procedures, recipients of KTx from living donors, and recipients of combined transplantations (eg, pancreas-kidney transplantation) will be excluded from the study. Eligible patients will be informed about the study protocol and will be asked to provide written informed consent to participate. Patients that agree to participate and sign the informed consent form (online supplemental file 2) will be enrolled and baseline demographic and clinical characteristics will be recorded. Reasons for exclusion from the PREFEN trial

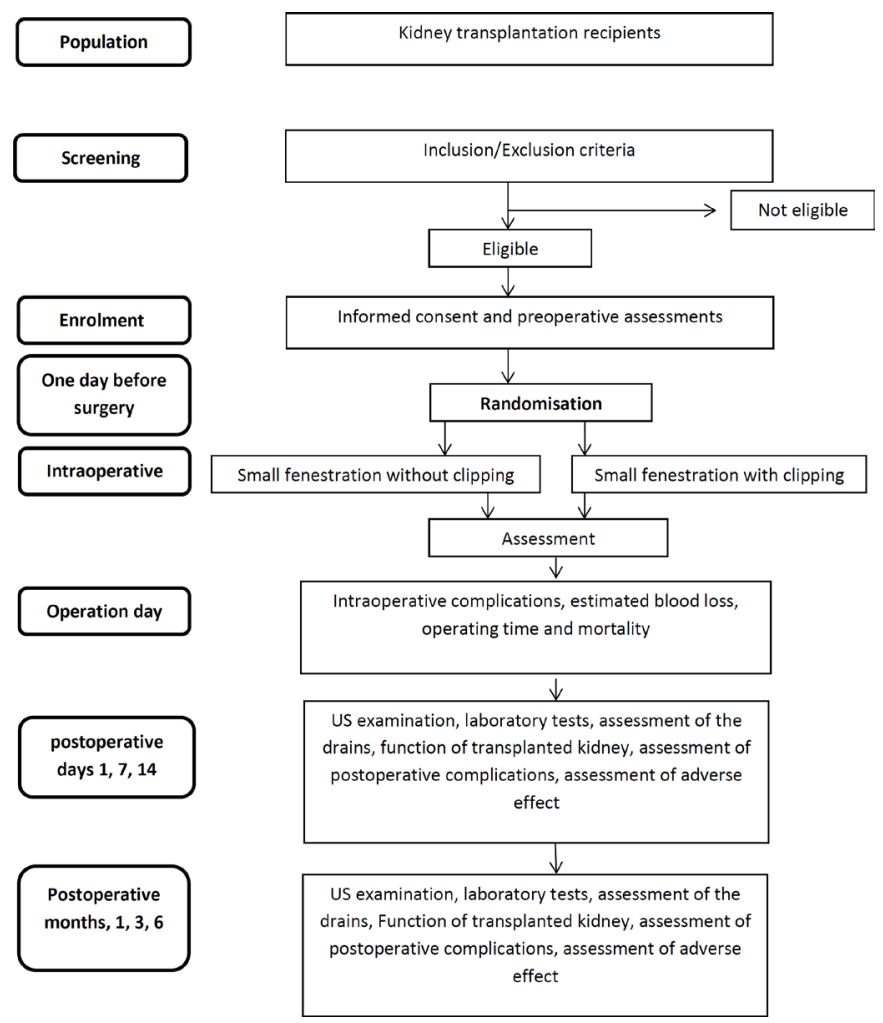

Figure 1 Study design flow chart.

will be documented and explained in the screening form. Included patients will be randomly assigned to one of the two study arms (small fenestration only or small fenestration with clipping of the edges).

\section{Outcome measures}

During the PREFEN trial, KTx recipients will be monitored before surgery, intraoperatively, on postoperative days (POD) 1, 7 and 14, or at discharge. After discharge, the recipients will be visited at postoperative months 1 , 3 and 6 , which are the routine postoperative follow-up appointments following KTx in our centre. To enhance participant retention and to avoid loss to follow-up, we will call the patients during the follow-up period to remind them of scheduled visits. If a patient is not able to participate in a follow-up visit, the appointment will be rescheduled by telephone. Ultrasound examinations will be performed on each visit as a routine clinical procedure, and fluid collections will be recorded. Demographic and baseline clinical data, intraoperative findings and postoperative results will be documented in case report forms (CRF) (table 1). Graft function will be assessed and documented at each follow-up visit.

\section{Primary endpoint}

The primary endpoint is the incidence of symptomatic post-KTx lymphocele and/or lymphorrhea, which requires interventional treatment in the 6 months following KTx. Depends on the PKTL location, these symptoms can be urological, vascular and/or visceral. So, compression on the urinary system may lead to obstruction of urine 
Table 1 PREFEN Study design according to the Standard Protocol Items: Recommendations for Interventional Trials checklist

\begin{tabular}{|c|c|c|c|c|c|c|c|c|}
\hline \multirow[b]{2}{*}{ Timepoint } & \multirow{2}{*}{$\begin{array}{l}\text { Enrolment } \\
\begin{array}{l}\text { Administration } \\
\text { day }\end{array}\end{array}$} & \multirow{2}{*}{$\begin{array}{l}\text { Intervention } \\
\text { Operation day }\end{array}$} & \multicolumn{5}{|c|}{ Post-allocation } & \multirow{2}{*}{$\begin{array}{r}\text { Close-ou } \\
\text { POM6 }\end{array}$} \\
\hline & & & POD1 & POD7 & POD14 & POM1 & РОМЗ & \\
\hline \multicolumn{9}{|l|}{ Enrolment } \\
\hline Eligibility screen & $\mathrm{x}$ & & & & & & & \\
\hline Informed consent & $x$ & & & & & & & \\
\hline Randomisation & $x$ & & & & & & & \\
\hline Allocation & $x$ & & & & & & & \\
\hline \multicolumn{9}{|l|}{ Interventions } \\
\hline $\begin{array}{l}\text { KTx } \\
\text { fenestration }\end{array}$ & & $x$ & & & & & & \\
\hline KTx fenestration and clipping & & $\mathrm{x}$ & & & & & & \\
\hline \multicolumn{9}{|l|}{ Assessment } \\
\hline Baseline assessment & $x$ & & & & & & & \\
\hline Intraoperative complications & & $\mathrm{x}$ & & & & & & \\
\hline Estimated blood loss & & $x$ & & & & & & \\
\hline Operating time & & $x$ & & & & & & \\
\hline $\begin{array}{l}\text { Post-operative } \\
\text { complications }^{*}\end{array}$ & & $\mathrm{x}$ & $x$ & $x$ & $x$ & $x$ & $x$ & $x$ \\
\hline Fluid collections & & $x$ & $x$ & $x$ & $x$ & $x$ & $x$ & $x$ \\
\hline Lymphocele formation & & $x$ & $x$ & $x$ & $x$ & $x$ & $x$ & $x$ \\
\hline Lymphorrhea & & $\mathrm{x}$ & $x$ & $x$ & $x$ & $x$ & $x$ & $x$ \\
\hline Length of hospital stay & & & $x$ & $x$ & $x$ & $x$ & $x$ & $x$ \\
\hline Mortality & & $x$ & $x$ & $x$ & $x$ & $x$ & $x$ & $x$ \\
\hline Primary non-function kidney & & & $x$ & $x$ & $x$ & $x$ & $x$ & $x$ \\
\hline Delayed graft function & & & $x$ & $x$ & $x$ & $x$ & $x$ & $x$ \\
\hline Creatinine level & $x$ & $x$ & $\mathrm{x}$ & $x$ & $x$ & $x$ & $x$ & $x$ \\
\hline BUN level & $x$ & $x$ & $x$ & $x$ & $x$ & $x$ & $x$ & $x$ \\
\hline Glomerular filtration rate & $x$ & $x$ & $\mathrm{x}$ & $x$ & $\mathrm{x}$ & $x$ & $x$ & $x$ \\
\hline Safety assessment & & $x$ & $x$ & $x$ & $x$ & $\mathrm{x}$ & $\mathrm{x}$ & $x$ \\
\hline
\end{tabular}

*Including fluid collections, burst abdomen, incisional hernia, intestinal herniation, wound infection, intra-abdominal bleeding/ haematoma, postoperative ileus and medical complications.

BUN, blood urea nitrogen; KTx, kidney transplantation; POD, postoperative day; POM, postoperative month.

outflow, urinary retention, dysuria, increased creatinine levels and/or graft dysfunction/failure. Potential vascular symptoms are mainly obstruction of transplant circulation, deep venous thrombosis, arterial hypertension and/or ipsilateral leg swelling. Visceral complications including bowel obstruction, organ displacement and/or wound dehiscence are also other possible symptoms. According to our classification of PKTL, post-KTx lymphorrhea is defined as lymph outflow of more than 50 $\mathrm{mL}$ fluid (not urine, blood or pus) per day from the drain or site of the removed drain, after the 7th post-KTx day. Analysis should not reveal any blood, pus or urine in the fluid. Post-KTx lymphocele is defined as a fluid collection near to the transplanted kidney in a non-epithelialised cavity (after urinoma, haematoma and abscess have been ruled out).
Demographic and baseline clinical data will be recorded for each recipient preoperatively (table 1). At each visit, results of routine laboratory patient assessments will be recorded and post-KTx fluid collection will also be assessed by ultrasound examination. During the ultrasound, the examiner will be blinded to the patient's randomisation arm. If fluid has collected at the site of implantation, the volume of hypoechoic perirenal fluid accumulation will be calculated. Perirenal fluid that is removed by aspiration, drainage or surgery will be analysed to rule out the other fluid collections. Additionally, the drain outflow will be assessed and analysed for post-KTx lymphorrhea after POD 7. After discharge, the patients will be followed up for 6 months. Recurrence is documented only in case of reappearance of lymphocele after intervention for treatment. Morbidities during 
Table 2 The definitions of the secondary endpoints

\begin{tabular}{|c|c|}
\hline Secondary endpoints & Definitions \\
\hline Warm ischaemia time & $\begin{array}{l}\text { The time (minutes) from clamping the first renal artery in situ to flushing the kidney } \\
\text { with chilled solution on the back table }\end{array}$ \\
\hline Intraoperative complications & Any complication occurring during the operation \\
\hline Estimated blood loss & The entire blood loss (millilitres) from skin incision to skin closure \\
\hline Operating time & The time (minutes) from skin incision to closure of the skin incision \\
\hline Postoperative complications & $\begin{array}{l}\text { Postoperative surgical complications (ie, fluid collections (seroma, haematoma, } \\
\text { intra-abdominal abscess or collection), burst abdomen, incisional hernia, } \\
\text { intestinal herniation, wound infection, intra-abdominal bleeding/haematoma and } \\
\text { postoperative ileus) and medical complications (pneumonia, pleural effusion and } \\
\text { urinary tract infection). Each complication will be graded according to the Clavien- } \\
\text { Dindo classification }{ }^{25}\end{array}$ \\
\hline Length of hospital stay & Time (days) from the day of the operation until the day of discharge \\
\hline Time to return to work & The number of days from discharge to return to work \\
\hline Incisional hernia & $\begin{array}{l}\text { Fascia or muscle defect (bulging hernial sac and palpable fascia gap) at the site of } \\
\text { the surgical incision examined by palpation and ultrasonography }\end{array}$ \\
\hline Mortality & Death due to any cause at any time during the follow-up period \\
\hline Primary non-function & The graft never functions \\
\hline Delayed graft function (DGF) & $\begin{array}{l}\text { The need for one or more haemodialysis treatments following transplantation before } \\
\text { the graft functions properly. The duration of DGF will be calculated from the date of } \\
\text { transplantation to the date of the last dialysis treatment }\end{array}$ \\
\hline Recipient serum creatinine level & Serum creatinine level (mg/dL) \\
\hline $\begin{array}{l}\text { Recipient serum blood urea nitrogen } \\
\text { (BUN) level }\end{array}$ & Serum BUN (mg/dL) \\
\hline Glomerular filtration rate (GFR) & GFR $\left(\mathrm{mL} / \mathrm{min} / 1.73 \mathrm{~m}^{2}\right)$ calculated as $175 \times\left(\mathrm{S}_{\mathrm{cr}}\right)^{-1.154} \times(\mathrm{Age})^{-0.203} \times(0.742$ if female $)$ \\
\hline
\end{tabular}

the 6-month follow-up and all-cause mortalities after 90 days will be recorded. Graft function will also be assessed by routine evaluation of serum creatinine, blood urea nitrogen (BUN) and glomerular filtration rate (GFR).

\section{Secondary endpoints}

Estimated blood loss, surgical complications and operation time will be reported intraoperatively. After the operation, duration of hospital stay, postoperative complications (including fluid collections, burst abdomen, incisional hernia, intestinal herniation, intraabdominal abscess or collection, and postoperative ileus) and all-cause mortality will be recorded for all patients. Incidence of total perirenal fluid accumulation, delayed graft function, serum creatinine levels, BUN level, GFR, primary non-function and delayed graft function will be documented at each follow-up visit. Secondary outcome measures are defined in table 2 .

\section{Standardised therapy and trial interventions}

All transplantations will be performed based on the standards of our centre. Recipients will be randomised into a control group (small fenestration without clipping) or an intervention group (small fenestration with clipping). For fenestration, a $2 \mathrm{~cm}$ round window will be made in the peritoneum, medial and close to the kidney hilum. One easy flow drain will be inserted through the peritoneal window into the abdomen and another easy flow drain will be placed parallel to the kidney in the retroperitoneal space. Both easy flow drains will be extracted through the abdominal wall and fixed to the skin. In the control group, the peritoneal edges will not be sutured and the edges will not be clipped. In the study group (small fenestration with clipping), the edges of the peritoneal window $\left(360^{\circ}\right)$ will be clipped with eight large metal clips (Horizon, Weck Closure Systems, Research Triangle Park, North Carolina, USA) in every $45^{\circ}$ in an oblique position (figure 2A,B). That means a total number of eight clips at $0^{\circ}, 45^{\circ}, 90^{\circ}, 135^{\circ}, 180^{\circ}, 225^{\circ}, 270^{\circ}$ and $315^{\circ}$.

\section{Patient and public involvement}

The patients and public were not involved in the planning of this study.

\section{Modification of the protocol}

If any modifications are necessary after patient recruitment starts, the amendments will be submitted to the ethics committee and no further patients will be recruited until the amendments are accepted.

\section{Assessment of safety and termination criteria}

The intervention-related events that occur during surgery and follow-up will be recorded. At each visit, the physician will ask the recipient if he/she has suffered from any serious adverse events (SAEs) since the last visit. The attending physician must inform the principal investigator 


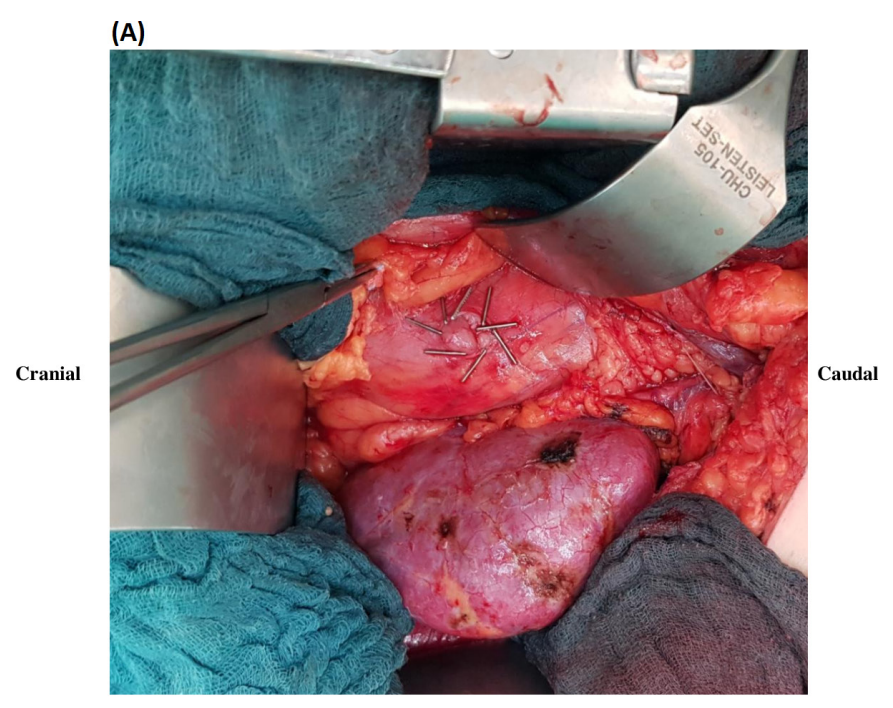

(B)

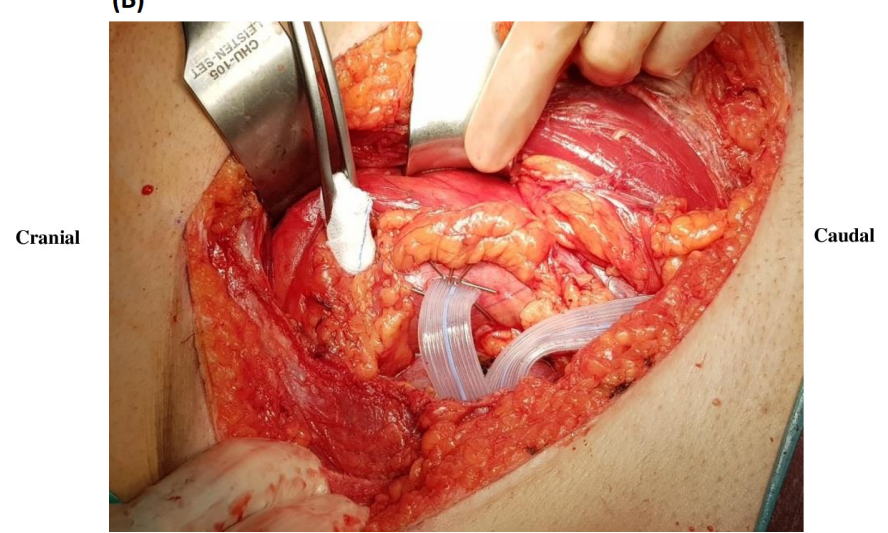

Figure 2 Clipping the fenestration edges, (A) without easy flow drain, and (B) with easy flow drain.

about any reported SAEs within 24 hours. The causes of completed and detailed SAEs will be investigated. If a study participant withdraws their consent to participate in the trial, they will be excluded from the study. Participants may withdraw their consent to participate at any time without explanation and without affecting further medical care. At any time during the study, an independent data safety monitoring committee may terminate the trial after consultation with the key research associates and the biostatistician. High morbidity or mortality rates and any indication of potential health hazards caused by either the study treatment or external factors are possible reasons for terminating the trial.

\section{Randomisation and blinding}

The 'block randomisation' method will be used to generate random sequences in this study. Each block will contain an equal number of participants in two groups: small fenestration without clipping and small fenestration with clipping. Possible balanced combinations of these groups within the block will be numbered consecutively. Then, blocks will be chosen randomly using simple randomisation software (Microsoft Excel). A series of randomly assigned preventive methods including 'small fenestration alone' and 'small fenestration with clipping' will be generated based on the random sequence of blocks. These allocations will be printed onto cards, which will then be sealed in sequentially numbered opaque envelopes. Blocks will be randomly chosen and participants will be assigned to the groups. Randomisation will be performed a day before the KTx operation by a university staff member, external to the research team, who is not involved in the operation, treatment of complications and assessment of patients.

Enrolled KTx patients will be randomly allocated to one of the two study arms the day before surgery. The trial executors will receive randomly generated treatment allocations within sealed opaque envelopes. At the beginning of the operation, the medical staff will personally inform the expert surgeon which treatment group the patient has been randomised to. To avoid any potential prediction of group allocation, information on the block length will not be kept at the study site.

During the postoperative follow-up, no information about the randomisation will be given to the treatment staff or the patient. The participant and/or assessor will only be unblinded in the case of medical emergencies or serious medical conditions that may not be adequately treated without unblinding. The primary and secondary endpoints of the trial will be documented. At the end of the trial, the data management centre will receive all sealed envelopes and will check the accuracy of randomisation numbers.

\section{Data management}

All data will be gathered and documented in paperbased CRFs by an investigator and then transferred to the data management centre. To assure precise data collection, CRF will be filled in by an investigator who is not involved in patient evaluation after each patient visit. All demographic and baseline clinical data and primary and secondary outcome measures will be recorded on the CRF. After rechecking all data, missing data will be obtained from the trial database or by reevaluating the study participants. Patient confidentiality is ensured by allocating an anonymous number to each patient's CRF. In the case of withdrawal from the study, permission to continue follow-up and data collection will be obtained. All completed CRFs will be reviewed and signed by the responsible investigator. All CRFs will be identifiable only by an anonymous number and stored in a locked filing cabinet at our clinic. These CRFs will be accessible only by the principal investigator. After all data are collected, the principal investigator of the study will transfer all the data to a locked Excel database. Only authorised and well-documented updates to the study data are possible after database lock. Afterwards, data stored in a locked Excel database will be statistically analysed by a statistician who is blinded to the allocated treatment for each patient. 


\section{Statistical methods}

Sample size

The focus of this exploratory trial is to generate preliminary data and to evaluate the outcomes following a small peritoneal fenestration with and without clipping. The number of patients required to obtain sufficient first data on efficacy and safety of the intervention has been estimated. It is planned to include 124 patients in this trial. Based on our experience, we expect a $10 \%$ loss to follow-up. The resulting sample size (62 in each group) should be enough to detect an incidence rate reduction of 5\% (which is the incidence of symptomatic PKTL required interventional treatment in the literature) to $1 \%$ (which is the incidence of lymphocele in an intraperitoneal situation ${ }^{2022}$ ) with $80 \%$ power and a significance level of 5\%, using Fisher's exact test. We believe that clipping the edges of a small fenestration will prevent early closure of the window, and reduce the rate of lymphocele formation without intestinal herniation or problems to perform a biopsy.

\section{Statistical analysis}

Statistical analysis will be performed based on the intention-to-treat populations using SPSS software 24.0 for Windows. The incidence and prevalence of categorical outcomes (lymphocele, lymphorrhea and fluid collection) will be presented as proportions and will be compared between groups using the $\mathrm{X}^{2}$ or Fisher's exact test. The risk ratio will be calculated to show the effect of small fenestration and clipping on the risk of lymphocele/lymphorrhea formation and other outcomes. Continuous data will be presented as means and SD and will be compared between the study groups using Student's t-test. Non-normally distributed continuous variables will be analysed using Wilcoxon rank-sum test. The Kaplan-Meier time-to-event method will be used for analysing the outcomes including length of hospital stay and time to return to work. Furthermore, to evaluate the impact of fenestration and clipping on the occurrence of post-KTx lymphocele in patients at high risk of lymphocele (obesity, use of mTOR inhibitors and history of graft rejection), subgroup analyses will be performed. The significance level will be set at $\alpha \leq 0.05$, representing $95 \%$ confidence.

\section{ETHICS AND DISSEMINATION \\ Ethical considerations}

This protocol study received approval from the Ethics Committee of the University of Heidelberg (Registration Number S-318/2017). A Standard Protocol Items: Recommendations for Interventional Trials checklist is available for this protocol (online supplemental file 3 ). The results will be disseminated through peer-reviewed journals and conference presentations.

Eligible patients will be informed about the study protocol and will be asked to provide written informed consent to participate. Patients that agree to participate will sign the informed consent. Patient confidentiality is ensured by allocating an anonymous number to each patient's CRF. In the case of withdrawal from the study, permission to continue follow-up and data collection will be obtained. All completed CRFs will be reviewed and signed by the responsible investigator. After the data are collected, they will be statistically analysed by a statistician who is blinded to the allocated treatment for each patient.

\section{Dissemination plan}

The results and findings of the trial will be published in peer-reviewed journals and presented at conferences in order to disseminate the results of this trial.

\section{DISCUSSION}

Peritoneal fenestration after KTx reduces the risk of perirenal fluid collection and symptomatic lymphocele formation. ${ }^{20}{ }^{23}$ Fenestration of the peritoneal wall and drainage of lymph into the peritoneal cavity effectively prevents post-KTx lymphocele formation by intraperitoneal absorption of lymph. ${ }^{3023}$ Only one clinical trial has compared the impact of preventive fenestration in KTx patients. Syversveen et $a l^{20}$ included $130 \mathrm{KTx}$ patients in their study and performed preventive fenestration in 69 randomly selected patients. In this study, the peritoneum was incised parallel to the skin incision and the incision was the length of the transplanted kidney or longer. The edges were not clipped in this study and lymphocele was diagnosed by ultrasound imaging during the 10 weeks after surgery. Their results showed that prophylactic fenestration reduces the risk of lymphocele formation after KTx and decreases the need for invasive treatment, however with higher rate of intestinal complications. ${ }^{20}$ Although Syversveen et al provided valuable information regarding preventive effect of peritoneal fenestration, the risk of intestinal herniation or difficulties for performing biopsies with such a large window are challenging. In this regard, some surgeons prefer to use smaller fenestrations to decrease the above mentioned complications. Previous studies have mentioned that lymphoceles recur after early closure of the peritoneal window. ${ }^{21}{ }^{24}$ This is more likely if a smaller peritoneal fenestration is used. Although the size of the window may not be the only important element in preventing closure, the window may close spontaneously if it is smaller than $3 \times 5 \mathrm{~cm} .{ }^{21}$ Window closure was avoided by clipping the window edges during laparoscopic therapeutic fenestration in a study from Taweemonkongsap et $a .^{21}$ In this study, the authors reported no recurrence of lymphoceles during a 40-month follow-up period after treatment of lymphocele. However, the preventive effect of this method has still not been investigated.

The PREFEN trial will be the first RCT to compare the impact of small fenestration alone, with small fenestration and clipping on preventing lymphoceles and lymphorrhea formation after KTx. Post-KTx lymphocele may affect graft function by putting pressure on the kidney or by compressing the ureter or transplant 
vasculature. In addition, ipsilateral leg oedema, genital oedema and deep vein thrombosis may also occur after compression of the external iliac vein. ${ }^{13}$ Peritoneal fenestration decreases the rate of PKTL, hospitalisation time and costs. Reoperations and invasive interventions after KTx have a negative impact on long-term graft survival. Reducing post-surgical complications will reduce the need for interventions following KTx, thereby improving the long-term outcome of KTx.

There are some limitations to the present RCT. Contrary to some centres, intraoperative easy flow drain will be placed in all participants of present RCT. Drain placement may affect the rate of post-KTx lymphatic complications, and thus can lead to different results compared with other centres. Furthermore, since performing fenestration results in peritoneal breach, postoperative peritoneal dialysis will not be possible in these patients. Therefore, patient selection should be performed cautiously, especially for patients, in whom haemodialysis is contraindicated.

In summary, although peritoneal fenestration reduces the rate of postoperative lymphatic complications, the size of the peritoneal window remains a challenging issue. A big peritoneal window results in herniation and difficulties in performing biopsies, meanwhile a small one will close early without sufficient effect. A method that can reduce the window size while keeping its effect would resolve this problem. The PREFEN trial is the first RCT to analyse the impact of clipping the window edges on optimising the effect of peritoneal fenestration in KTx and reducing fenestration-related complications. The findings of this study may help to reduce the rate of graft failure, hospitalisation time and costs.

Contributors AM and MG developed the original concept of the trial. AM, MG, MS, EK and OG developed the design and methodology. EK, OG, MS and SM performed the statistical assessments and developed the analysis plan. MS, EK, OG, SM, MAS and MG contributed to drafting the protocol of the paper and the article. MM, ALM, $M D, C M, M Z$, YK and AM contributed to the revision of the final report and study protocol. All authors read and approved the final manuscript.

Funding The authors have not declared a specific grant for this research from any funding agency in the public, commercial or not-for-profit sectors.

Competing interests None declared.

Patient consent for publication Obtained.

Provenance and peer review Not commissioned; externally peer reviewed.

Open access This is an open access article distributed in accordance with the Creative Commons Attribution Non Commercial (CC BY-NC 4.0) license, which permits others to distribute, remix, adapt, build upon this work non-commercially, and license their derivative works on different terms, provided the original work is properly cited, appropriate credit is given, any changes made indicated, and the use is non-commercial. See: http://creativecommons.org/licenses/by-nc/4.0/.

ORCID iD

Arianeb Mehrabi http://orcid.org/0000-0001-6163-1525

\section{REFERENCES}

1 Mihaljevic AL, Heger P, Abbasi Dezfouli S, et al. Prophylaxis of lymphocele formation after kidney transplantation via peritonea fenestration: a systematic review. Transpl Int 2017;30:543-55.

2 Ranghino A, Segoloni GP, Lasaponara F, et al. Lymphatic disorders after renal transplantation: new insights for an old complication. Clin Kidney J 2015;8:615-22.

3 Golriz M, Klauss M, Zeier M, et al. Prevention and management of lymphocele formation following kidney transplantation. Transplant Rev 2017;31:100-5.

4 Guerrero-Ramos F, Cavero-Escribano T, Rodríguez-Antolín A, et al. Perigraft fluid collections after kidney transplantation: does the type of donor (uncontrolled donation after circulatory death vs. donation after brain death) have a role? Cent European J Urol 2017;70:439-44.

5 Mehrabi A, Kashfi A, Tönshoff B, et al. Long-Term results of paediatric kidney transplantation at the University of Heidelberg: a 35 year single-centre experience. Nephrol Dial Transplant 2004;19 Suppl 4:iv69-74.

6 Lucewicz A, Wong G, Lam VWT, et al. Management of primary symptomatic lymphocele after kidney transplantation: a systematic review. Transplantation 2011;92:663-73.

7 Presser N, Kerr H, Gao T, et al. Fibrin glue injections: a minimally invasive and cost-effective treatment for Post-Renal transplant Lymphoceles and lymph fistulas. Am J Transplant 2016;16:694-9.

8 Derweesh IH, Ismail HR, Goldfarb DA, et al. Intraoperative placing of drains decreases the incidence of lymphocele and deep vein thrombosis after renal transplantation. BJU Int 2008;101:1415-9.

9 Tammaro V, Vernillo A, Dumani X, et al. Prevention of fluid effusion in kidney transplantation with the use of hemostatic biomaterials. Transplant Proc 2014;46:2203-6.

10 Berardinelli L, Raiteri M, Pasciucco A, et al. The use of a polymeric sealant for prevention of posttransplantation lymphocele. Transplant Proc 2011;43:1072-3.

11 Chandrasekaran D, Meyyappan RM, Rajaraman T. Instillation of povidone iodine to treat and prevent lymphocele after renal transplantation. BJU Int 2003;91:296.

12 Nowak K, Bönninghoff R, Geiger M, et al. Compression stockings limit the incidence of postoperative lymphocele in kidney transplantation. In Vivo 2013;27:561-4.

13 letto G, Amico F, Soldini G, et al. Real-Time intraoperative fluorescent lymphography: a new technique for lymphatic sparing surgery. Transplant Proc 2016;48:3073-8.

14 Simforoosh N, Tabibi A, Rad HM, et al. Comparison between bipolar lymphatic vessels cautery and suture ligature in prevention of Postrenal transplant lymphocele formation: a randomized controlled trial. Exp Clin Transplant 2019;17:26-30.

15 Sidebottom RC, Parsikia A, Chang P-N, et al. No benefit when placing drains after kidney transplant: a complex statistical analysis. Exp Clin Transplant 2014;12:106-12.

16 Bischof G, Rockenschaub S, Berlakovich G, et al. Management of lymphoceles after kidney transplantation. Transpl Int 1998;11:277-80.

17 Lee HS, Joo DJ, Huh KH, et al. Laparoscopic fenestration versus percutaneous catheter drainage for lymphocele treatment after kidney transplantation. Transplant Proc 2013;45:1667-70.

18 Lima MLde, Cotrim CAC, Moro JC, et al. Laparoscopic treatment of lymphoceles after renal transplantation. Int Braz J Urol 2012;38:215-21.

19 Ulrich F, Niedzwiecki S, Fikatas P, et al. Symptomatic lymphoceles after kidney transplantation - multivariate analysis of risk factors and outcome after laparoscopic fenestration. Clin Transplant 2010;24:273-80.

20 Syversveen T, Midtvedt K, Brabrand K, et al. Prophylactic peritoneal fenestration to prevent morbidity after kidney transplantation: a randomized study. Transplantation 2011;92:196-202.

21 Taweemonkongsap T, Srinualnad S, Nualyong C, et al. Novel technique to prevent lymphocele recurrence after laparoscopic lymphocele fenestration in renal transplant patients. J Endourol 2006;20:654-8.

22 Ebadzadeh MR, Tavakkoli M. Lymphocele after kidney transplantation: where are we standing now? Urol J 2008;5:144-8.

23 Layman RE, McNally M, Kilian C, et al. Does opening the peritoneum at the time of renal transplanation prevent lymphocele formation? Transplant Proc 2006;38:3524-6.

24 Bailey SH, Mone MC, Holman JM, et al. Laparoscopic treatment of post renal transplant lymphoceles. Surg Endosc 2003;17:1896-9.

25 Dindo D, Demartines N, Clavien P-A. Classification of surgical complications: a new proposal with evaluation in a cohort of 6336 patients and results of a survey. Ann Surg 2004;240:205-13. 